\title{
Improvement in Cheyne-Stokes respiration following cardiac resynchronisation therapy
}

\author{
J.Y. Gabor, D.A. Newman, V. Barnard-Roberts, V. Korley, I. Mangat, \\ P. Dorian and P.J. Hanly
}

ABSTRACT: The effect of standard cardiac resynchronisation therapy (CRT) on the severity of Cheyne-Stokes respiration (CSR) in patients with congestive heart failure was studied. It was hypothesised that CRT, through its known beneficial effects on cardiac function, would stabilise the control of breathing and reduce CSR.

Twenty-eight patients who were eligible for CRT and receiving optimised medical treatment for congestive heart failure were referred for overnight polysomnography, including monitoring of thoracic and abdominal movements to identify CSR and obstructive sleep apnoea events. Patients underwent repeat polysomnography after 6 months of CRT to re-evaluate sleep quality and sleepdisordered breathing.

Twelve of the 28 patients had significant CSR (43\%); 10 patients had a successful implantation and underwent repeat polysomnography a mean $\pm S D 27 \pm 7$ weeks after continuous biventricular pacing. Six of the 10 patients experienced a significant decrease in CSR severity following CRT, associated with correction of congestive heart failure-related hyperventilation and hypocapnia. Circulation time, oxygen saturation, frequency of obstructive apnoeas and sleep quality did not change.

In conclusion, cardiac resynchronisation therapy is associated with a reduction in CheyneStokes respiration, which may contribute to improved clinical outcome in patients treated with cardiac resynchronisation therapy.

KEYWORDS: Cardiac resynchronisation therapy, Cheyne-Stokes respiration

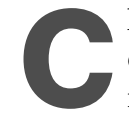
heyne-Stokes respiration (CSR) is a form of periodic breathing defined by recurrent episodes of central apnoeas or hypopnoeas, alternating with hyperpnoeas, which exhibit a crescendo-decrescendo pattern of tidal volume [1, 2]. CSR is characteristically associated with congestive heart failure (CHF), and has a reported prevalence of $40-50 \%$ in patients whose left ventricular ejection fraction is $<40 \%$ [3-5]. Most CHF patients with CSR chronically hyperventilate due to stimulation of pulmonary vagal irritant receptors from pulmonary venous congestion secondary to left ventricular (LV) dysfunction [6, 7] and enhanced central and peripheral chemosensitivity $[8,9]$. Prolonged circulation time, due to impaired LV function, does not contribute to the initiation of CSR, but does influence the length of the periodic breathing cycle by delaying transmission of changes in arterial blood gas tensions from the lungs to the chemoreceptors [10, 11].

Previous studies have demonstrated an association between the presence of CSR and increased mortality in CHF [12], which is independent of other potentially confounding risk factors, suggesting that CSR accelerates the deterioration in cardiac function, perhaps through the augmentation of sympathetic nervous system activity, haemodynamic fluctuations or the induction of ventricular arrhythmias [13-16]. If CSR does alter the natural history of $\mathrm{CHF}$, then the presence of CSR may be an indication to intensify CHF therapy, treat CSR independently or consider novel therapies that reduce CSR severity.

Numerous studies have suggested that shortand long-term cardiac resynchronisation therapy (CRT) can improve clinical CHF symptoms, cardiac function, quality of life, and functional capacity [17-22]. CRT has been shown to lower pulmonary capillary wedge pressure [17], decrease mitral regurgitation [22], and increase cardiac output. All of these effects should reduce pulmonary oedema and the tendency to hyperventilate, thereby stabilising the control of breathing. Therefore, the current authors wanted to determine, using objective and standard measures, if CRT is associated with a reduction in CSR severity in patients with $\mathrm{CHF}$.
AFFILIATIONS

Dept of Medicine, St. Michael's Hospital, University of Toronto, Toronto, Canada.

CORRESPONDENCE

P.J. Hanly

1421 Health Sciences Center 3330 Hospital Drive NW

Calgary $\mathrm{AB}$

T2N 4N1

Canada

Fax: 14032836151

E-mail: phanly@ucalgary.ca

Received

August 102004

Accepted after revision:

March 072005 


\section{METHODS}

\section{Study protocol}

Consecutive patients with chronic CHF (New York Heart Association (NYHA) functional class II, III or IV), who were accepted for CRT by a cardiologist (D.A. Newman, P. Dorian, I. Mangat or V. Korley), were referred for evaluation of sleepdisordered breathing. The selection of these patients was based on their suitability for CRT and was not based on the presence of sleep or respiratory symptoms. No patients were receiving treatment for sleep apnoea prior to enrolment or during the course of the study. Patients were recruited between November 1999 and October 2002. Standard overnight polysomnography (PSG) was performed within the week prior to implant, including monitoring of thoracic and abdominal movements to identify central and obstructive apnoeas. Each patient's heart failure medications were optimised and remained stable for $\geqslant 3$ months prior to implant, and were not altered during the study period. Patients identified with CSR had repeat PSG following $\sim 6$ months (mean \pm SD $27 \pm 7$ weeks) of stable biventricular pacing. Left ventricular ejection fraction (LVEF), left ventricular end-diastolic dimension (LVEDD) and the severity of mitral regurgitation were determined by two-dimensional Doppler-flow echocardiography, both at baseline and after 6 months of biventricular pacing. Subjective assessment of daytime sleepiness was assessed by the administration of a validated questionnaire, the Epworth Sleepiness Scale [23], on the night of each sleep study.

\section{Polysomnography}

Comprehensive overnight PSG was performed in all subjects using Sandman 5.0 software (NPB-Melville, Ottawa, Canada). The recording montage included the following: two-channel electroencephalography (C3-A2, C4-A1); electrooculography; submental and bilateral anterior tibialis electromyography; electrocardiography; measurements of thoracic and abdominal respiratory efforts by respiratory inductance plethysmography (Respitrace $\left.{ }^{(}\right)$; Ambulatory Monitoring Inc., White Plains, NY, USA); finger pulse oximetry; and transcutaneous capnography (Kontron Medical; Hoffman-LaRoche, Basel, Switzerland). Obstructive apnoeas/hypopnoeas were defined by the absence/reduction of tidal volume for $\geqslant 10 \mathrm{~s}$, associated with paradoxical thoraco-abdominal motion. Central apnoeas/ hypopnoeas were defined by the absence/reduction of tidal volume for $\geqslant 10 \mathrm{~s}$ with an absence of thoracic and abdominal excursions. CSR was defined as repetitive episodes of central apnoea or hypopnoea, alternating with hyperpnoea, with a characteristic crescendo-decrescendo pattern [24]. The apnoea-hypopnoea (AHI) index was defined as the number of apnoeas and hypopnoeas per hour of sleep. All studies were interpreted accorded to standard criteria [25] by registered PSG technologists blinded to cardiac function test results. A patient with $>10$ CSR events per hour of sleep was considered to have CSR. CSR cycle length was calculated as previously described [10], using an average of 10 randomly selected CSR events during non-rapid eye movement (REM) sleep. Lung-tofinger circulation time (LFCT) was calculated as previously described [26], measured on the same CSR events as those used to determine cycle length. In the case of patients whose CSR index dropped to zero following CRT, obstructive apnoeas were used to calculate cycle length and LFCT.
Total sleep time (TST) was defined as the number of hours asleep in any sleep stage, as defined by standard PSG criteria [25]. Sleep efficiency, sleep latency, and wakefulness after sleep onset were defined as described previously [27]. Arousals and awakenings from sleep were defined by standard criteria [28], classified into one of the three following types: 1) respiratory arousal (apnoea or hypopnoea within the preceding $3 \mathrm{~s}$ ); 2) periodic limb movement (PLM) arousal (a PLM [29] within the preceding $3 \mathrm{~s}$ ); or 3 ) spontaneous arousal (i.e. not temporally related to either a respiratory event or a PLM).

\section{Biventricular pacing}

CHF patients eligible for CRT met the following criteria: 1) heart failure (NYHA functional class II, III or IV) secondary to idiopathic or ischaemic left ventricular systolic dysfunction; 2) LVEF <35\%; 3) LVEDD > $55 \mathrm{~mm}$; 4) QRS interval > $130 \mathrm{~ms}$; 5) receiving appropriate and optimised treatment for heart failure, including a diuretic, an angiotensin-converting enzyme inhibitor and/or an angiotensin-receptor blocker, and a betablocker. Patients with unstable angina, myocardial infarction, cerebrovascular accident or transient ischaemic attack within the previous 3 months, severe primary pulmonary disease or haemodynamic instability (systolic blood pressure $<80 \mathrm{mmHg}$ or $>170 \mathrm{mmHg}$ ) were excluded from the resynchronisation protocol. Eligible patients underwent implantation of a cardiac resynchronisation device with three pacing leads: a standard right atrial lead; a standard right ventricular lead; and a specialised left ventricular lead, which was placed into a typical posterolateral coronary vein. Post-implant programming was standardised for all patients.

\section{Statistical analysis}

All data are reported as mean $\pm \mathrm{SD}$. Paired t-tests were used to compare variables pre- and post-resynchronisation therapy. Unpaired t-tests were used to compare continuous data between two independent groups. One-way ANOVA, with post hoc Bonferroni tests, was used to compare continuous data between multiple groups. Chi-squared tests were used to compare categorical data. Mann-Whitney rank-sum tests were used to compare ordinal data between two independent groups. Wilcoxon signed-rank tests were used to compare ordinal paired data. A p-value of $<0.05$ was considered to be statistically significant.

\section{RESULTS}

\section{Subject demographics}

A total of $28 \mathrm{CHF}$ patients (22 males, six females) underwent baseline PSG during the study period. Of these patients, 12 ( $43 \%$; seven males, five females) had no significant apnoea of any type (central AHI $<10$ and obstructive AHI $<10$ ), four (14\%; three males, one female) had obstructive sleep apnoea (OSA; mean \pm SD obstructive AHI $20.7 \pm 13.7$ ), and the remaining 12 males (43\%) had CSR (central AHI 30.8 \pm 14.9 ). Of these 12 patients, one patient's implantation was unsuccessful and a second patient declined repeat PSG, and, consequently, these patients were not followed further. Three of the remaining 10 CSR patients also had significant coexisting OSA (obstructive AHI 29.1 \pm 6.1 ). No significant differences existed with respect to age, body mass index (BMI) or baseline cardiac function between patient subgroups with or without sleep apnoea and CSR ( $p>0.05$; table 1). Patients with sleep apnoea were 


\begin{tabular}{|c|c|c|c|c|}
\hline \multirow[t]{2}{*}{ TABLE 1} & \multicolumn{4}{|c|}{$\begin{array}{l}\text { Patient characteristics prior to cardiac } \\
\text { resynchronisation therapy }\end{array}$} \\
\hline & Total & Non-SA & SA & CSR \\
\hline Subjects n & 28 & 12 & 16 & 12 \\
\hline Age yrs & $62.8 \pm 10.7$ & $60.1 \pm 8.1$ & $64.8 \pm 12.1$ & $65.1 \pm 13.7$ \\
\hline BMI $\mathbf{k g} \cdot \mathrm{m}^{-2}$ & $28.6 \pm 3.2$ & $28.4 \pm 2.6$ & $28.9 \pm 3.7$ & $28.6 \pm 3.8$ \\
\hline Males/females* & 22/6 & $7 / 5$ & $15 / 1$ & $12 / 0$ \\
\hline ICM/non-ICM & $11 / 17$ & $4 / 8$ & $7 / 9$ & $5 / 7$ \\
\hline LVEF \% & $19.8 \pm 3.2$ & $20.4 \pm 3.0$ & $19.3 \pm 3.4$ & $19.8 \pm 4.1$ \\
\hline QRS ms & $177 \pm 22$ & $170 \pm 23$ & $181 \pm 20$ & $187 \pm 22.7$ \\
\hline NYHA II/III/IV" & $3 / 19 / 6$ & $1 / 11 / 0$ & $2 / 8 / 6$ & $1 / 6 / 5$ \\
\hline LVEDD cm & $6.91 \pm 0.77$ & $6.90 \pm 0.89$ & $6.92 \pm 0.71$ & $7.23 \pm 0.75$ \\
\hline 6-min walk m & $354 \pm 64$ & $357 \pm 54$ & $351 \pm 80$ & $342 \pm 84$ \\
\hline \multicolumn{5}{|c|}{$\begin{array}{l}\text { Data are presented as } n \text { and mean } \pm \text { SD. SA: sleep apnoea (includes patients } \\
\text { with obstructive sleep apnoea and Cheyne-Stokes respiration (CSR)); BMI: } \\
\text { body mass index; ICM: ischaemic cardiomyopathy; LVEF: left ventricular } \\
\text { ejection fraction; NYHA: New York Heart Association; LVEDD: left ventricular } \\
\text { end-diastolic dimension. *: } p<0.05 \text { between SA and non-SA, } p<0.05 \text { between } \\
\text { CSR and non-SA; \#: } p<0.05 \text { between CSR and non-SA. }\end{array}$} \\
\hline
\end{tabular}

predominantly male $(\mathrm{p}<0.05)$ and had a worse NHYA classification $(\mathrm{p}<0.05)$.

\section{Sleep quality}

At baseline, all $10 \mathrm{CSR}$ patients who completed the study reported at least one symptom of poor sleep, such as a history of snoring $(n=6)$, witnessed apnoea during sleep $(n=5)$, nocturnal choking $(n=4)$, waking unrefreshed $(n=5)$ and impairment of memory and concentration $(n=5)$. Only two patients reported falling asleep unintentionally during the day. Baseline PSG indicated that patients suffered sleep loss, reflected by a shortened total sleep time and reduced sleep efficiency (table 2). Sleep loss was due both to a difficulty in initiating sleep, reflected by a prolonged sleep latency, and difficulty maintaining sleep, reflected by prolonged wakefulness after sleep onset (WASO). Furthermore, sleep quality was compromised, as evidenced by an increased proportion of stage-1 non-REM sleep and a reduced proportion of REM sleep compared with historical controls [30]. Given the high frequency of observed arousals and awakenings, reduced sleep quality in this group was probably attributable to sleep fragmentation. The majority of arousals were categorised as "respiratory arousals", indicating that they were induced by episodes of CSR.

Sleep was more disrupted in patients with CSR than those without sleep apnoea, reflected by a higher proportion of stage-1 non-REM sleep and a higher frequency of arousals related to apnoeas and hypopnoeas (table 2). However, sleep quantity, as measured by TST, sleep efficiency, WASO and sleep latency, did not change following 6 months of CRT. Furthermore, there were no changes in any PSG parameter of sleep quality, such as sleep architecture and arousal frequencies $(\mathrm{p}>0.05)$. Patients did report subjective improvements in sleep quality during follow-up clinic examination, such as requiring less time in bed to awaken refreshed, diminished requirements for daytime naps, a reduction in perceived

\begin{tabular}{|c|c|c|c|}
\hline \multirow[t]{2}{*}{ TABLE 2} & $\begin{array}{l}\text { phy follow } \\
\text { ion therap }\end{array}$ & $\begin{array}{l}\text { g cardiac } \\
\text { (CRT) }\end{array}$ & \multirow[b]{2}{*}{$\begin{array}{c}\text { Post-CRT } \\
\text { CSR } \\
\text { patients }\end{array}$} \\
\hline & $\begin{array}{c}\text { Pre-CRT } \\
\text { non-SA } \\
\text { patients }\end{array}$ & $\begin{array}{c}\text { Pre-CRT } \\
\text { CSR } \\
\text { patients }\end{array}$ & \\
\hline Subjects $n$ & 12 & 10 & 10 \\
\hline TST h & $5.1 \pm 0.9$ & $4.6 \pm 1.5$ & $5.0 \pm 1.1$ \\
\hline Sleep efficiency \% & $74.4 \pm 12.5$ & $65.1 \pm 21.1$ & $69.2 \pm 13.4$ \\
\hline Sleep latency min & $23.2 \pm 28.5$ & $13.1 \pm 8.3$ & $27.2 \pm 24.9$ \\
\hline WASO min & $83 \pm 40$ & $135 \pm 91$ & $104 \pm 52$ \\
\hline Stage $1 \% \mathrm{TST}^{*}$ & $8 \pm 9$ & $20 \pm 13$ & $21 \pm 15$ \\
\hline Stage $2 \%$ TST & $62 \pm 13$ & $58 \pm 9$ & $59 \pm 11$ \\
\hline SWS \% TST & $18 \pm 11$ & $9 \pm 7$ & $6 \pm 6$ \\
\hline REM sleep \% TST & $12 \pm 7$ & $13 \pm 7$ & $14 \pm 7$ \\
\hline PLM index $\cdot h^{-1}$ & $36 \pm 41$ & $15 \pm 24$ & $27 \pm 38$ \\
\hline PLM arousal index $\cdot h^{-1}$ & $9 \pm 13$ & $3 \pm 4$ & $6 \pm 9$ \\
\hline Respiratory arousal index $\cdot h^{-1}$ * & $2 \pm 2$ & $26 \pm 9$ & $25 \pm 16$ \\
\hline Spontaneous arousal index $\cdot h^{-1}$ & $5 \pm 3$ & $4 \pm 4$ & $5 \pm 6$ \\
\hline Total arousal index $\cdot h^{-1 \star}$ & $15 \pm 16$ & $32 \pm 11$ & $36 \pm 13$ \\
\hline Awakening index $\cdot \mathrm{h}^{-1}$ & $6 \pm 5$ & $12 \pm 10$ & $10 \pm 6$ \\
\hline
\end{tabular}

Data are presented as mean \pm SD. SA: sleep apnoea (includes patients with obstructive sleep apnoea and Cheyne-Stokes respiration (CSR)); TST: total sleep time; WASO: wakefulness after sleep onset; SWS: slow-wave sleep; REM: rapid eye movement; PLM: periodic limb movement. ${ }^{*}: p<0.05$ between nonSA patients and CSR patients.

daytime sleepiness and an increase in energy. However, this subjective improvement in sleep quality and daytime function was not reflected in the Epworth Sleepiness Scale, which did not change significantly $(p=0.26)$.

\section{Improvement in CSR following CRT}

The severity of CSR decreased significantly ( $<<0.05$; table 3 ) as measured by the number of central apnoeas and hypopnoeas per hour of sleep. In the three CSR patients who also had significant OSA (obstructive AHI $>15$ ), the obstructive AHI decreased in two patients and increased in one; overall, the change was not significant $(25.8 \pm 10.4$ to $13.8 \pm 12.0 ; \mathrm{p}>0.05)$. BMI and percentage of TST spent in the supine position, variables that could both affect the AHI, did not change from baseline to follow-up (BMI: $28.9 \pm 4.2$ versus $29.4 \pm 4.5, \mathrm{p}>0.05$; $\%$ TST supine: $29.9 \pm 26.3$ versus $32.6 \pm 32.2, \mathrm{p}>0.05)$. Mixed apnoeas, which have features of both central and obstructive events, were few and did not change significantly following CRT ( $2.6 \pm 4.1$ versus $4.1 \pm 8.4 ; \mathrm{p}>0.05)$. Consequently, reclassification of mixed apnoeas to "obstructive" or "central" events would not have altered the present results. Although the severity of CSR in the patient cohort decreased following CRT, four of the 10 CSR patients did not experience a decrease (central AHI: $27.5 \pm 13.2$ versus $32.6 \pm 10.8 ; \mathrm{p}>0.05$ ), in contrast to the six patients who did (central AHI: $32.7 \pm 15.3$ to $3.8 \pm 4.4$; $\mathrm{p}<0.05$; fig. 1). Transcutaneous partial pressure of carbon dioxide $\left(\mathrm{PCO}_{2}\right)$ did not change significantly $(\mathrm{p}>0.05)$; however, in the six patients whose CSR improved following CRT, the increase in mean transcutaneous $\mathrm{PCO}_{2}$ during sleep correlated with the observed reduction in central AHI $(p=0.07 ; \mathrm{r}=0.85$; 


\begin{tabular}{|c|c|c|}
\hline & Pre-CRT & Post-CRT \\
\hline Subjects n & 10 & 10 \\
\hline Total AHI $\cdot h^{-1}$ & $42.7 \pm 9.1$ & $30.8 \pm 18.7$ \\
\hline CSR events $\cdot h^{-1 *}$ & $30.6 \pm 14.0$ & $15.3 \pm 16.5$ \\
\hline Obstructive $A H I \cdot h^{-1}$ & $9.7 \pm 12.3$ & $11.8 \pm 10.2$ \\
\hline Mean $\mathrm{Sa}, \mathrm{O}_{2}$,awake \% & $93.6 \pm 1.0$ & $93.9 \pm 0.7$ \\
\hline Mean $\mathrm{Sa}, \mathrm{O}_{2}$, sleep $\%$ & $93.3 \pm 1.1$ & $93.3 \pm 1.1$ \\
\hline Mean $\mathrm{PCO}_{2}, \mathrm{tc}$,awake $\mathrm{mmHg}$ & $42.1 \pm 4.1$ & $43.6 \pm 4.9$ \\
\hline Mean $\mathrm{PCO}_{2}, \mathrm{tc}$,sleep $\mathrm{mmHg}$ & $42.2 \pm 3.9$ & $44.9 \pm 4.4$ \\
\hline PB cycle length s & $63.6 \pm 16.2$ & $59.1 \pm 9.6$ \\
\hline LFCT s & $43.0 \pm 12.4$ & $35.6 \pm 6.8^{\#}$ \\
\hline \multicolumn{3}{|c|}{$\begin{array}{l}\text { Data are presented as mean } \pm \mathrm{SD} \text {, unless otherwise stated. AHI: apnoea- } \\
\text { hypopnoea index; CSR: Cheyne-Stokes respiration; } \mathrm{Sa}_{\mathrm{a}} \mathrm{O}_{2} \text { : arterial oxyger } \\
\text { saturation; } \mathrm{PCO}_{2} \text {,tc: transcutaneous partial pressure of carbon dioxide; PB } \\
\text { periodic breathing; } \mathrm{LFCT} \text { : lung-to-finger circulation time. } \bullet: \text { includes mixec } \\
\text { apnoeas in addition to CSR and obstructive apnoeas. }{ }^{*}: \mathrm{p}<0.05 \text { versus pre } \\
\mathrm{CRT} \text {; : } \mathrm{p}=0.09 \text {. }\end{array}$} \\
\hline
\end{tabular}

$\left.r^{2}=0.73\right)$. Mean oxygen saturation did not change following CRT either during sleep or when awake $(p>0.05)$. Periodic breathing cycle length did not change $(p>0.05)$. LFCT tended to change following CRT, in the entire group $(p=0.09)$ and in the six patients whose CSR improved $(\mathrm{p}=0.08)$. No patient without CSR prior to CRT developed any sleep-disordered breathing following CRT. Six patients had non-ischaemic cardiomyopathy (ICM) and four had ICM. There was no difference between these two subgroups in terms of improvement in CSR or in the change in cardiac function following CRT.

\section{Cardiac function following CRT}

Patients experienced a significant improvement in LVEF, LV grade, NYHA class, severity of mitral regurgitation and 6-min walk $(\mathrm{p}<0.05$; table 4$)$. LVEDD did not change significantly

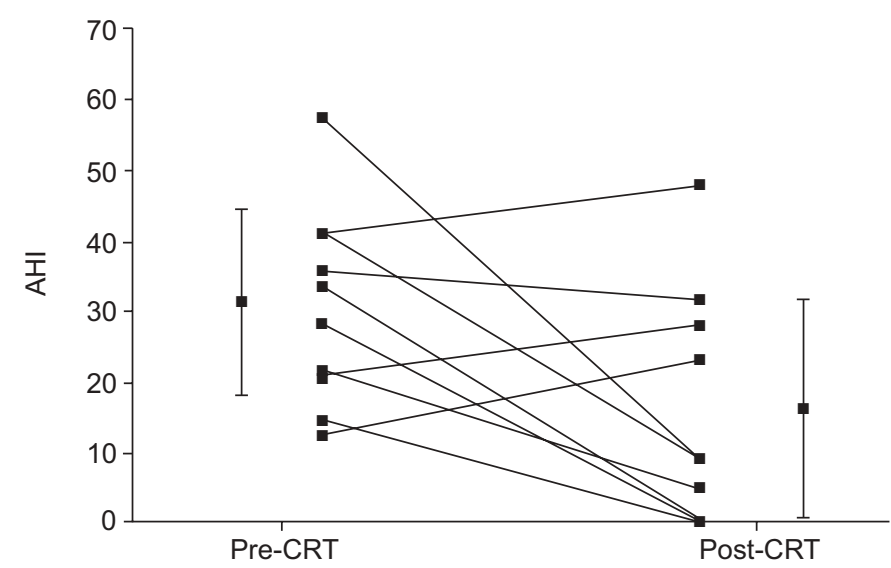

FIGURE 1. Change in central apnoea-hypopnoea index (AHI) following 6 months of cardiac resynchronisation therapy (CRT) in Cheyne-Stokes respiration patients. Data are expressed as mean $\pm \mathrm{SD}$.

\section{TABLE 4 Cardiac function before and 6 months after cardiac resynchronisation therapy (CRT)}

\begin{tabular}{lcc} 
& Pre-CRT & Post-CRT \\
\hline Subjects $\mathbf{n}$ & 10 & 10 \\
LVEF* \% & $19.0 \pm 4.2$ & $24.2 \pm 7.8$ \\
LV grade* 2/3/4 & $0 / 3 / 7$ & $1 / 5 / 3$ \\
NYHA class* $\mathbf{~ / I I / I I I / I V ~}$ & $0 / 1 / 7 / 2$ & $2 / 6 / 2 / 0$ \\
6-min walk* & $333 \pm 84$ & $376 \pm 100$ \\
LVEDD cm & $7.25 \pm 0.71$ & $7.06 \pm 0.95$ \\
Mitral regurgitation* mild/mod./severe & $3 / 4 / 3$ & $8 / 2 / 0$ \\
Mean heart rate awake & $68 \pm 8$ & $67 \pm 12$ \\
Max. heart rate awake & $92 \pm 18$ & $88 \pm 18$ \\
Min. heart rate awake & $46 \pm 10$ & $50 \pm 7$ \\
Mean heart rate sleep & $66 \pm 9$ & $66 \pm 12$ \\
Min. heart rate sleep & $47 \pm 7$ & $50 \pm 12$ \\
Max. heart rate sleep & $83 \pm 15$ & $82 \pm 14$ \\
\hline
\end{tabular}

Data are presented as $\mathrm{n}$ or mean \pm SD. LVEF: left ventricular ejection fraction; LV: left ventricle; NYHA: New York Heart Association; LVEDD: left ventricular end-diastolic dimension; mod.: moderate; max.: maximum; min.: minimum. * $\mathrm{p}<0.05$ versus pre-CRT.

$(\mathrm{p}>0.05)$. Mean, minimum or maximum heart rate both when awake and during sleep did not change significantly $(\mathrm{p}>0.05)$. In the subgroup of four patients whose CSR did not improve following CRT, there was no significant change in LVEF $(20 \pm 6.5$ to $28 \pm 8.6 \% ; p=0.165)$, LV grade, mitral regurgitation or the 6-min walk test $(279 \pm 66$ to $310 \pm 77 \mathrm{~min} ; \mathrm{p}=0.079)$, but there was a significant improvement in LVEDD $(7 \pm 0.68$ to $6 \pm 0.86 \mathrm{~cm} ; \mathrm{p}=0.04)$ and NYHA class improved by one level in each patient. Patients whose CSR improved had a greater baseline 6-min walk $(383 \pm 67$ versus $268 \pm 69 ; \mathrm{p}<0.05)$ than patients whose CSR did not improve, and, post-CRT, tended to have milder mitral regurgitation $(p=0.07)$ and a better NYHA class $(p=0.05)$. All patients reported subjective improvements in CHF symptoms, such as energy, exercise tolerance and reduced dyspnoea. No patients required hospitalisation for exacerbation of CHF symptoms during the study period.

\section{DISCUSSION}

The current data indicate that, in a cohort of heart failure patients referred for CRT, patients have poor sleep quality (reported subjectively and determined objectively), associated with a high prevalence of sleep apnoea, predominantly in the form of CSR. The addition of CRT to maximum medical therapy improved the severity of CSR in the majority of patients. Therefore, CRT may represent an additional therapy for CSR in CHF patients.

\section{Sleep quality}

CSR patients reported subjective sleep disruption and symptoms such as snoring, waking unrefreshed, and impairment of memory and concentration. Objective assessment of sleep quality by overnight PSG revealed that these patients suffered both sleep loss and sleep fragmentation. This was mainly due to arousals and awakenings associated with sleep-disordered breathing, predominantly in the form of CSR. Although CRT 
improved CSR in the majority of patients, sleep quality continued to be poor due to persistent sleep disruption both from OSA and PLM. It may also have been related to the effects of cardiac medication and to conditioned insomnia associated with the disruptive effects of chronic heart failure over many years.

\section{Prevalence of sleep-disordered breathing}

In accordance with previous studies on CHF populations [3-5], the prevalence of CSR in this group is $\sim 40 \%$, with CSR patients more likely to be male and have a worse NYHA functional classification for an equivalent LVEF compared with their nonapnoeic counterparts. The prevalence of CSR was high, despite the fact that the patients were receiving optimal medical therapy and were not selected on the basis of suspected sleep apnoea. Whether the difference in NYHA class is a consequence of CSR or simply an indicator of poorer outcome remains to be determined.

\section{Impact of CRT on sleep-disordered breathing}

The most significant finding of the current study was a notable reduction in the severity of CSR, which occurred in two-thirds of patients. OSA, when present, did not significantly change. The lack of a consistent effect of CRT on obstructive apnoea severity was responsible for the persistent elevation of respiratory arousal frequency, which probably contributed to sleep disruption, since the majority of arousals were related to respiratory events. The coexistence of OSA and CSR in CHF patients has been previously documented [26], and the "unmasking" of OSA as CSR disappears may indicate the existence of a spectrum of sleep-disordered breathing, whose particular manifestation (CSR or OSA) depends on the underlying degree of cardiac dysfunction.

It was observed that the increase in mean transcutaneous $\mathrm{PCO}_{2}$ correlated with the reduction in central AHI in those patients whose CSR improved following CRT. These results support the work of previous authors [9], who demonstrated an inverse relationship between carbon dioxide arterial tension and pulmonary capillary wedge pressure, and that CHF patients with CSR have higher pulmonary capillary wedge pressures than those without CSR. This implies that hypocapnia and CSR are a manifestation of pulmonary venous congestion. Despite the small sample size in the current study, there was a significant improvement in several parameters of cardiac function following CRT (table 4). In addition, a trend towards decreased LFCT was observed, both in the entire group and in the patients whose CSR improved, which is most probably a further indication of an improvement in cardiac function. Consequently, the current authors feel that the most likely explanation for the improvement in CSR following CRT was that improved cardiac function reduced pulmonary venous congestion and associated hyperventilation during sleep.

\section{Limitations and comparisons with previous data}

The current study had an uncontrolled and observational design, which limited the authors' ability to attribute the improvement in cardiac function and CSR solely to CRT. The present authors tried to minimise this potential weakness in the study design by ensuring that all patients were on maximal medical therapy for $\mathrm{CHF}$ for $\geqslant 3$ months prior to enrolment and that their cardiac medication did not change throughout the study. Furthermore, cardiologists at the current authors' institution accepted patients for CRT only after their cardiac status had been stabilised on optimal therapy and without expectation of further improvement on medical therapy. Consequently, it is believed that the improvement in cardiac function was due to CRT rather than other factors, such as a delayed response to cardiac medication. The present study is also limited by a small sample size, which precludes very thorough statistical analyses. Furthermore, the analysis was not able to determine why CSR did not improve in some of the study patients, and, therefore, the current authors cannot predict which CHF patients with CSR may benefit most from CRT.

The only other study to examine the effect of CRT on CSR differed methodologically from the present study, in that the present study used comprehensive overnight, attended PSG, which monitored sleep, respiration, heart rate, PLM and body position, whereas the previous study used an ambulatory, nonattended, cardiorespiratory polygraph, which monitored respiration and heart rate alone [31, 32], and has not been validated in patients with CSR. It is believed that PSG provides a more accurate estimation of apnoea frequency, reliably distinguishes obstructive and central events, which are likely to coexist in patients with heart failure, in addition to objectively monitoring sleep quality and identifying PLM. Furthermore, the present study included measurements of circulation time and CSR cycle length, and employed a longer mean duration to follow-up (27 weeks versus 17 weeks). The current authors' patient demographics were similar to those of SINHA et al. [31], although three of the present 10 subjects had NYHA class IV heart failure prior to CRT; two of these three did not show an improvement in CSR. By contrast, all of the patients of SINHA et al. [31] were NYHA class III. Although the current authors' results are more equivocal than those of SINHA et al. [31], they are nonetheless positive, and emphasise the need to clearly define the heart failure population that best responds to CRT.

\section{Conclusions}

Cardiac resynchronisation therapy results in an improvement in cardiac function, consistent with previous studies, and reduces the frequency of Cheyne-Stokes respiration in some patients. Further investigations are required to determine the mechanisms by which cardiac resynchronisation therapy corrects Cheyne-Stokes respiration and which clinical variables best predict a favourable response in this regard.

\section{ACKNOWLEDGEMENTS}

The authors wish to sincerely thank S. Thorne, M. Sharma and J. Beecroft for their extensive administrative and logistical assistance during the study.

\section{REFERENCES}

1 Cheyne J. A case of hypoplexy, in which the fleshy part of the heart was converted into fat. Dublin Hosp Rep 1818; 2: 216-223.

2 Stokes W. The Diseases of the Heart and the Aorta. Dublin, Hodges and Smith, 1854. 
3 Javaheri S, Parker TJ, Wexler L, et al. Occult sleepdisordered breathing in stable congestive heart failure. Ann Intern Med 1995; 122: 487-492.

4 Sin DD, Fitzgerald F, Parker JD, Newton G, Floras JS, Bradley TD. Risk factors for central and obstructive sleep apnea in 450 men and women with congestive heart failure. Am J Respir Crit Care Med 1999; 160: 1101-1106.

5 Javaheri S, Parker TJ, Liming JD, et al. Sleep apnea in 81 ambulatory male patients with stable heart failure. Types and their prevalences, consequences, and presentations. Circulation 1998; 97: 2154-2159.

6 Solin P, Bergin P, Richardson M, Kaye DM, Walters EH, Naughton MT. Influence of pulmonary capillary wedge pressure on central apnea in heart failure. Circulation 1999; 99: 1574-1579.

7 Lorenzi-Filho G, Azevedo ER, Parker JD, et al. Relationship of carbon dioxide tension in arterial blood to pulmonary wedge pressure in heart failure. Eur Respir J 2002; 19: 37-40.

8 Javaheri S. A mechanism of central sleep apnea in patients with heart failure. N Engl J Med 1999; 341: 949-954.

9 Solin P, Roebuck T, Johns DP, Walters EH, Naughton MT. Peripheral and central ventilatory responses in central sleep apnea with and without congestive heart failure. Am J Respir Crit Care Med 2000; 162: 2194-2200.

10 Naughton M, Benard D, Tam A, Rutherford R, Bradley TD. Role of hyperventilation in the pathogenesis of central sleep apneas in patients with congestive heart failure. Am Rev Respir Dis 1993; 148: 330-338.

11 Hall MJ, Xie A, Rutherford R, Ando S, Floras JS, Bradley TD. Cycle length of periodic breathing in patients with and without heart failure. Am J Respir Crit Care Med 1996; 154: 376-381.

12 Hanly PJ, Zuberi-Khokhar N. Increased mortality associated with Cheyne-Stokes respiration in patients with congestive heart failure. Am J Respir Crit Care Med 1996; 153: 272-276.

13 Trinder J, Merson R, Rosenberg JI, Fitzgerald F, Kleiman J, Douglas Bradley T. Pathophysiological interactions of ventilation, arousals, and blood pressure oscillations during Cheyne-Stokes respiration in patients with heart failure. Am J Respir Crit Care Med 2000; 162: 808-813.

14 Lorenzi-Filho G, Dajani HR, Leung RS, Floras JS, Bradley TD. Entrainment of blood pressure and heart rate oscillations by periodic breathing. Am J Respir Crit Care Med 1999; 159: 1147-1154.

15 Naughton MT, Benard DC, Liu PP, Rutherford R, Rankin F, Bradley TD. Effects of nasal CPAP on sympathetic activity in patients with heart failure and central sleep apnea. Am J Respir Crit Care Med 1995; 152: 473-479.

16 Javaheri S, Corbett WS. Association of low $\mathrm{PaCO}_{2}$ with central sleep apnea and ventricular arrhythmias in ambulatory patients with stable heart failure. Ann Intern Med 1998; 128: 204-207.

17 Leclercq C, Cazeau S, Le Breton H, et al. Acute hemodynamic effects of biventricular DDD pacing in patients with end-stage heart failure. I Am Coll Cardiol 1998; 32: 1825-1831.

18 Auricchio A, Stellbrink C, Block M, et al. Effect of pacing chamber and atrioventricular delay on acute systolic function of paced patients with congestive heart failure. The Pacing Therapies for Congestive Heart Failure Study Group. The Guidant Congestive Heart Failure Research Group. Circulation 1999; 99: 2993-3001.

19 Etienne Y, Mansourati J, Touiza A, et al. Evaluation of left ventricular function and mitral regurgitation during left ventricular-based pacing in patients with heart failure. Eur J Heart Fail 2001; 3: 441-447.

20 Cazeau S, Leclercq C, Lavergne T, et al. Effects of multisite biventricular pacing in patients with heart failure and intraventricular conduction delay. N Engl J Med 2001; 344: 873-880.

21 Abraham WT, Fisher WG, Smith AL, et al. Cardiac resynchronization in chronic heart failure. $N$ Engl J Med 2002; 346: 1845-1853.

22 Breithardt OA, Sinha AM, Schwammenthal E, et al. Acute effects of cardiac resynchronization therapy on functional mitral regurgitation in advanced systolic heart failure. $J$ Am Coll Cardiol 2003; 41: 765-770.

23 Johns MW. A new method for measuring daytime sleepiness: the Epworth sleepiness scale. Sleep 1991; 14: 540-545.

24 Brown HW, Plum F. The neurologic basis of CheyneStokes respiration. Am J Med 1961; 30: 849-860.

25 Rechtschaffen A, Kales A. A manual of standardized terminology, techniques and scoring system for sleep stages of human subjects (NIH Publication No. 2040). Los Angeles, Brain Information Service/Brain Research Institute UCLA, 1968.

26 Tkacova R, Niroumand M, Lorenzi-Filho G, et al. Overnight shift from obstructive to central apneas in patients with heart failure: role of $\mathrm{PCO}_{2}$ and circulatory delay. Circulation 2001; 103: 238-243.

27 Cooper AB, Thornley KS, Young GB, Slutsky AS, Stewart TE, Hanly PJ. Sleep in critically ill patients requiring mechanical ventilation. Chest 2000; 117: 809-818.

28 Mathur R, Douglas NJ. Frequency of EEG arousals from nocturnal sleep in normal subjects. Sleep 1995; 18: 330-333.

29 American Sleep Disorders Association. The international classification of sleep disorders: diagnostic and coding manual. Lawrence, KS, Allen Press, 1990; pp. 65-68.

30 Carskadon M, Dement W. Normal human sleep: an overview. In: Kryger $\mathrm{MH}$, Roth $\mathrm{T}$, Dement $\mathrm{WC}$, eds. Principles and Practice of Sleep Medicine. 2nd Edn. Philadelphia, WB Saunders Company, 1994; pp. 16-25.

31 Sinha AM, Skobel EC, Breithardt OA, et al. Cardiac resynchronization therapy improves central sleep apnea and Cheyne-Stokes respiration in patients with chronic heart failure. J Am Coll Cardiol 2004; 44: 68-71.

32 Ficker JH, Weist GH, Wilpert J, Fuchs FS, Hahn EG. Evaluation of a portable recording device (Somnocheck) for use in patients with suspected obstructive sleep apnea. Respiration 2001; 68: 307-312. 